\title{
RECEIVE KNOWLEDGE ONLY POWER ALLOCATION FOR NONREGENERATIVE COOPERATIVE MIMO COMMUNICATION
}

\author{
Fabien Héliot, Sadegh Fazel, Reza Hoshyar, and Rahim Tafazolli \\ Centre for Communication Systems Research, FEPS, University of Surrey, \\ Guildford GU2 7XH, UK, Email:\{F.Heliot, M.Fazel, R.Hoshyar, R.Tafazolli\}@ surrey.ac.uk
}

\begin{abstract}
Amplify-and-forward (AF) is one of the most popular approaches to transmit information over a cooperative multiinput multi-output (MIMO) relay channel. In AF, relay simply amplifies and retransmits the received signal from the source by relying only on the receive channel state information (CSI) available at the relay. In this paper we propose two novel power allocation methods for nonregenerative cooperative communication, assuming as in original AF that only receive CSI is available at the relay. Both methods have been designed to maximize the mutual information of the relay link: the first method by using an analytical solution and the second by solving a concave optimization problem. Performance of both our methods have been compared against traditional AF power allocation techniques in a MIMO environment, and results have demonstrated the efficiency of these methods in terms of mutual information and bit-error rate.
\end{abstract}

\section{INTRODUCTION}

Cooperative communication has recently attracted considerable research interests [1-6]. In a simple cooperation scenario composed of a source $(\mathrm{S})$ node, a single relay $(\mathrm{R})$ node and a destination (D) node, three main links are established, i.e., S-D, S-R, and R-D links. Various approaches have been followed to design cooperative communication systems, the most notorious ones are decode and forward (DF) and amplify and forward (AF) $[1-3,6]$. DF is a regenerative approach where the full decoding of the source message followed by the forwarding of the whole message to the destination node via the relay node are performed. On the contrary, AF is a simple nonregenerative approach where the relay node amplifies and forwards the received signal from the $\mathrm{S}$ node.

In cooperative multi-input multi-output (MIMO) scenario, instead of using the relay like a simple equal gain (EG) amplifier as it is done in the original AF scheme, it can be utilized as a smart precoder, which allows mutual information improvement through efficient power allocation techniques based on the level of CSI available at the R node. In the

This work has been performed in the framework of the FP7 project ROCKET IST-215282 STP, which is funded by the European Community. case that both the CSI of the S-R and R-D links are known at the $\mathrm{R}$ node, power allocation techniques have been recently developed $[7,8]$ and they have shown to greatly enhance the performance of AF in terms of mutual information. However, these techniques required extra complexity since they rely not only on S-R link CSI as in original AF, but as well as on R-D link CSI, which may not always be available. Here, we also aim at improving the performance of $\mathrm{AF}$ but without requiring extra CSI knowledge, as in the AF matched filter based relaying (MFR) and AF minimum mean square error filtering (MMSEF) techniques which have been lately proposed in [8].

In this paper, we design two novel power allocation methods for nonregenerative cooperative MIMO system, relying on the system model introduced in Section 2. First, in Section 3 , we propose an analytical power allocation method based on the maximization of the expectation of the relay link mutual information. Then, we introduce a novel power allocation algorithm based on the maximization of the relay link mutual information considering equal eigen mode (EEM). The first method provides close-to-optimum mutual information performance but with a high implementation complexity, whereas the second method is low-complexity and still outperforms traditional EG and other existing power allocation techniques such as MFR and MMSEF, both in terms of mutual information and bit-error rate (BER), as it is reported in Section 4. Finally, conclusions are drawn in Section 5.

\section{CLASSIC COOPERATIVE MIMO COMMUNICATION SYSTEM MODEL}

We consider a classic cooperative MIMO communication system composed of three nodes, where a $\mathrm{S}$ node equipped with $n$ antennas cooperates with a nonregenerative $\mathrm{R}$ node equipped with $q$ antennas to transmit data to a D node equipped with $r$ antennas, as depicted in Fig. 1.

For the simplicity of the introduction, we assume a half duplex relaying scenario with two equal duration phases as in $[7,8]$, where in the first phase the BS broadcasts the signal $x$ to $D$ and $R$, and in the second phase only $R$ transmits to $\mathrm{D}$. During the first phase, the signal $\mathrm{x}$ is received as $\mathbf{y}_{0}=\mathbf{H}_{0} \mathbf{x}+\mathbf{n}_{0}$ and $\mathbf{y}_{1}=\mathbf{H}_{1} \mathbf{x}+\mathbf{n}_{1}$ at the $\mathrm{D}$ and $\mathrm{R}$, respectively, where $\mathbf{H}_{0} \in \mathbb{C}^{r \times n}$ and $\mathbf{H}_{1} \in \mathbb{C}^{q \times n}$ characterize the 


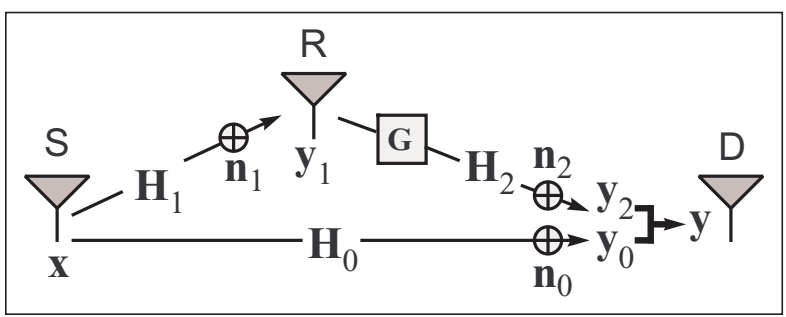

Fig. 1. Nonregenerative cooperative MIMO communication system model.

MIMO channel between the S-D and S-R links, correspondingly. During the second phase, the signal $\mathbf{y}_{1}$ is modified by using the precoding matrix $\mathbf{G} \in \mathbb{R}^{q \times q}$, then is transmitted towards D and is received as $\mathbf{y}_{2}=\mathbf{H}_{2} \mathbf{G y}_{1}+\mathbf{n}_{2}$, where $\mathbf{H}_{2} \in \mathbb{C}^{r \times q}$ characterizes the MIMO channel between the R$\mathrm{D}$ link. Moreover $\mathbf{n}_{0} \in \mathbb{C}^{r \times 1}, \mathbf{n}_{1} \in \mathbb{C}^{q \times 1}$ and $\mathbf{n}_{2} \in \mathbb{C}^{r \times 1}$ are vectors of independent zero-mean complex Gaussian noise entries with a variance of $\sigma^{2}$. The system model of the cooperative MIMO communication system introduced in Fig. 1 can be summarized as follows

$$
\mathbf{y}=\left[\begin{array}{l}
\mathbf{y}_{0} \\
\mathbf{y}_{2}
\end{array}\right]=\left[\begin{array}{c}
\mathbf{H}_{0} \\
\mathbf{H}_{2} \mathbf{G H}_{1}
\end{array}\right] \mathbf{x}+\left[\begin{array}{ccc}
\mathbf{I}_{r} & \mathbf{0} & \mathbf{0} \\
\mathbf{0} & \mathbf{H}_{2} \mathbf{G} & \mathbf{I}_{r}
\end{array}\right]\left[\begin{array}{l}
\mathbf{n}_{0} \\
\mathbf{n}_{1} \\
\mathbf{n}_{2}
\end{array}\right],
$$

with $\mathbf{I}_{r}$ is a $r \times r$ identity matrix. Consequently, the aggregate mutual information of the cooperative communication system in Fig. 1 can be expressed as [9]

$$
I(\mathbf{y} ; \mathbf{x})=\frac{1}{2} \log _{2}\left|\mathbf{I}_{2 r}+\mathbf{H R}_{x} \mathbf{H}^{\dagger} \mathbf{R}_{\mathbf{n}}^{-1}\right|,
$$

where

$\mathbf{H}=\left[\begin{array}{c}\mathbf{H}_{0} \\ \mathbf{H}_{2} \mathbf{G H}_{1}\end{array}\right], \mathbf{R}_{\mathbf{n}}=\left[\begin{array}{cc}\mathbf{R}_{\mathbf{n}_{0}} & \mathbf{0} \\ \mathbf{0} & \mathbf{H}_{2} \mathbf{G R}_{\mathbf{n}_{1}} \mathbf{G}^{\dagger} \mathbf{H}_{2}^{\dagger}+\mathbf{R}_{\mathbf{n}_{2}}\end{array}\right]$,

$\mathbf{H}^{\dagger}$ denotes the conjugate transpose of $\mathbf{H}, \mathbf{R}_{x}=\mathrm{E}\left\{\mathbf{x} \mathbf{x}^{\dagger}\right\}$ is the transmit signal covariance matrix, $\mathbf{R}_{\mathbf{n}_{0}}, \mathbf{R}_{\mathbf{n}_{1}}$ and $\mathbf{R}_{\mathbf{n}_{2}}$ are noise covariance matrices. Notice that the factor $1 / 2$ in (2) accounts for the 2-phases transmission.

Recently in [7], the aggregate mutual information $I(\mathbf{y} ; \mathbf{x})$ has been shown to be bounded as $I\left(\mathbf{y}_{0} ; \mathbf{x}\right)+I\left(\mathbf{y}_{2} ; \mathbf{x}\right) \geq$ $I(\mathbf{y} ; \mathbf{x}) \geq I\left(\mathbf{y}_{2} ; \mathbf{x}\right)$, where $I\left(\mathbf{y}_{0} ; \mathbf{x}\right)$ is the mutual information of the direct link and $I\left(\mathbf{y}_{2} ; \mathbf{x}\right)$ is the mutual information of the relay link given by

$$
\begin{aligned}
& I\left(\mathbf{y}_{0} ; \mathbf{x}\right)=\frac{1}{2} \log _{2}\left|\mathbf{I}_{2 k q}+\mathbf{H}_{0} \mathbf{R}_{x} \mathbf{H}_{0}^{\dagger} \mathbf{R}_{\mathbf{n}_{0}}^{-1}\right|, \\
& I\left(\mathbf{y}_{2} ; \mathbf{x}\right)=\frac{1}{2} \log _{2}\left|\frac{\mathbf{R}_{\mathbf{n}_{2}}+\mathbf{H}_{2} \mathbf{G}\left(\mathbf{R}_{\mathbf{n}_{1}}+\mathbf{H}_{1} \mathbf{R}_{x} \mathbf{H}_{1}^{\dagger}\right) \mathbf{G}^{\dagger} \mathbf{H}_{2}^{\dagger}}{\mathbf{R}_{\mathbf{n}_{2}}+\mathbf{H}_{2} \mathbf{G} \mathbf{R}_{\mathbf{n}_{1}} \mathbf{G}^{\dagger} \mathbf{H}_{2}^{\dagger}}\right|,
\end{aligned}
$$

respectively. Hence, the aggregate mutual information $I(\mathbf{y} ; \mathbf{x})$ can be increased by maximizing $I\left(\mathbf{y}_{2} ; \mathbf{x}\right)$ in $(3)$, which means by optimizing $\mathbf{G}$ at the $\mathrm{R}$ node, when $\mathbf{H}_{0}$ is unknown.

\section{NOVEL RELAY RECEIVE KNOWLEDGE ONLY POWER ALLOCATION METHODS}

Here, we first introduce in Section 3.1 the common methods to design the precoding matrix $\mathbf{G}$ when only S-R link CSI is available at the $\mathrm{R}$ node. Then, we introduce our novel power allocation methods based on the maximization of $I\left(\mathbf{y}_{2} ; \mathbf{x}\right)$ in Sections 3.2 and 3.3. In the following, we assume $\mathbf{R}_{\mathbf{n}_{0}}=$ $\mathbf{R}_{\mathbf{n}_{2}}=\sigma^{2} \mathbf{I}_{r}$ and $\mathbf{R}_{\mathbf{n}_{1}}=\sigma^{2} \mathbf{I}_{q}$, with $\sigma=1$.

\subsection{Traditional AF power allocation methods}

In original $\mathrm{AF}$, the precoding matrix $\mathbf{G}$ design follows an $\mathrm{EG}$ approach. In other words, $\mathbf{G}$ is designed such that the same amount of power is transmitted on any of the available eigen mode of the R-D link, and such that the total transmit power of the R node, i.e., $P_{2}$, respects the following constraint

$$
P_{\mathrm{R}}: \mathrm{E}\left\{\left\|\mathbf{G}\left(\mathbf{H}_{1} \mathbf{x}+\mathbf{n}_{1}\right)\right\|_{F}^{2}\right\} \leq P_{2},
$$

where $\mathrm{E}\{$.$\} is the expectation. Consequently, in the AF EG$ approach, $\mathbf{G}$ can be expressed as follows

$$
\mathbf{G}=\sqrt{P_{2}} \mathbf{J}\left(\mathrm{E}\left\{\left\|\mathbf{J}\left(\mathbf{H}_{1} \mathbf{x}+\mathbf{n}_{1}\right)\right\|_{F}^{2}\right\}\right)^{-\frac{1}{2}},
$$

with $\mathbf{J}=\mathbf{I}_{q}$. Lately in [8], other AF based approaches denoted MFR and MMSEF have also been proposed. They rely on the same $\mathbf{G}$ matrix formulation as in (5), but where $\mathbf{J}=\mathbf{H}_{1}^{\dagger}$ and $\mathbf{J}=\left(P_{1} / n\right) \mathbf{H}_{1}^{\dagger}\left[\mathbf{I}_{q}+\left(P_{1} / n\right) \mathbf{H}_{1} \mathbf{H}_{1}^{\dagger}\right]^{-1}$, respectively, and $P_{1}$ is the total transmit power of the $\mathrm{S}$ node.

\subsection{Analytical power allocation method}

We propose here a novel analytical power allocation method to optimize $\mathbf{G}$ based on the expectation of $I\left(\mathbf{y}_{2} ; \mathbf{x}\right)$ over $\mathbf{H}_{2}$. In the case that $\mathbf{H}_{1}$ is known at the $\mathrm{R}$ node, $\mathbf{H}_{1}$ can be decomposed via singular valued decomposition (SVD) as $\mathbf{H}_{1}=$ $\mathbf{U}_{1} \widehat{\Lambda}_{1}^{\frac{1}{2}} \mathbf{V}_{1}^{\dagger}$ where $\mathbf{U}_{1} \in \mathbb{C}^{q \times q}$ and $\mathbf{V}_{1} \in \mathbb{C}^{n \times n}$ are unitary matrices, and $\widehat{\boldsymbol{\Lambda}}_{1}$ is a $q \times n$ rectangular diagonal matrix. Moreover, we consider that $\mathbf{R}_{x}=\left(P_{1} / n\right) \mathbf{I}_{n}$. Consequently, $I\left(\mathbf{y}_{2} ; \mathbf{x}\right)$ in (3) can be re-expressed as $I\left(\mathbf{y}_{2} ; \mathbf{x}\right)=$

$$
\frac{1}{2} \log _{2}\left|\mathbf{I}_{r}+\mathbf{H}_{2} \widetilde{\mathbf{F}} \widetilde{\mathbf{F}}^{\dagger} \mathbf{H}_{2}^{\dagger}\right|-\frac{1}{2} \log _{2}\left|\mathbf{I}_{r}+\mathbf{H}_{2} \widetilde{\mathbf{G}} \widetilde{\mathbf{G}}^{\dagger} \mathbf{H}_{2}^{\dagger}\right|,
$$

where $\widetilde{\mathbf{G}}=\mathbf{G} \mathbf{U}_{1}=\operatorname{diag}\left(\sqrt{p_{2,1}}, \sqrt{p_{2,2}}, \ldots, \sqrt{p_{2, q}}\right)$ is a $q \times q$ diagonal matrix and $\widetilde{\mathbf{F}}=\widetilde{\mathbf{G}}\left(\mathbf{I}_{q}+\left(P_{1} / n\right) \boldsymbol{\Lambda}_{1}\right)$. Furthermore, $\boldsymbol{\Lambda}_{1}=\widehat{\boldsymbol{\Lambda}}_{1}^{\frac{1}{2}} \widehat{\boldsymbol{\Lambda}}_{1}^{\frac{1}{2} \dagger}$ is a $q \times q$ diagonal matrix with diagonal elements $\lambda_{1, i} \in \mathbb{C}$, which are sorted in descending order. Notice that $\lambda_{1, i} \neq 0$ for $i \in\left[1, L_{1}\right]$ and that $\lambda_{1, i}=0$ for $i \in\left[L_{1}+1, q\right]$, with $L_{1}=\min \{q, n\}$. Recently in [10], we have shown that the expression $\mathrm{E}\left\{\log _{2}\left|\mathbf{I}_{r}+\mathbf{H} \boldsymbol{\Delta} \mathbf{H}^{\dagger}\right|\right\}_{\mathbf{H}}$ is asymptotically equivalent to

$$
\chi(\delta)=\frac{1}{\ln (2)}\left[\ln \left(\frac{r^{r} \prod_{i=1}^{q}\left(d_{0} \delta_{i}+1\right)}{d_{0}^{r}}\right)-\sum_{i=1}^{q} \frac{d_{0} \delta_{i}}{d_{0} \delta_{i}+1}\right]_{(7)}
$$


for any $\mathbf{H} \in \mathbb{C}^{r \times q}$ and large values of $r$ and $q$, where $\boldsymbol{\Delta}=$ $\operatorname{diag}(\delta)$ is a $q \times q$ diagonal matrix, $\delta=\left\{\delta_{1}, \delta_{2}, \ldots, \delta_{q}\right\}$, and $d_{0}$ is the only nonnegative root of the polynomial given by

$$
P(d)=(d-r) \prod_{i=1}^{q}\left(\frac{1}{\delta_{i}}+d\right)+d \sum_{i=1}^{q} \prod_{\substack{j=1 \\ j \neq i}}^{q}\left(\frac{1}{\delta_{j}}+d\right) .
$$

Notice that (7) is similar to (19) in [10], for $n=1, m=q$, $\alpha=1, \beta=r, \omega=1$, and $v_{i}=1 / \delta_{i}$ in [10]. Then by solving the following concave optimization problem

$$
\begin{aligned}
& \max _{\mathbf{p}_{2}} \frac{1}{2}\left[\chi\left(\mathbf{p}_{2}\left(\mathbf{I}_{q}+\left(P_{1} / n\right) \boldsymbol{\Lambda}_{1}\right)\right)-\chi\left(\mathbf{p}_{2}\right)\right] \\
& \text { s.t. } p_{2, i} \geq 0 ; P_{R}: \sum_{i=1}^{L_{1}} p_{2, i}\left(1+\left(P_{1} / n\right) \lambda_{1, i}\right) \leq P_{2},
\end{aligned}
$$

where $\mathbf{p}_{2}=\left\{p_{2,1}, p_{2,2}, \ldots, p_{2, q}\right\}$, we obtain the $p_{2, i}$ values that maximize $\mathrm{E}\left\{I\left(\mathbf{y}_{2} ; \mathbf{x}\right)\right\}_{\mathbf{H}_{2}}$. The following problem cannot be easily solved by applying classic concave/convex optimization tools [11]. However, it can be solved by using a recursive function as follows

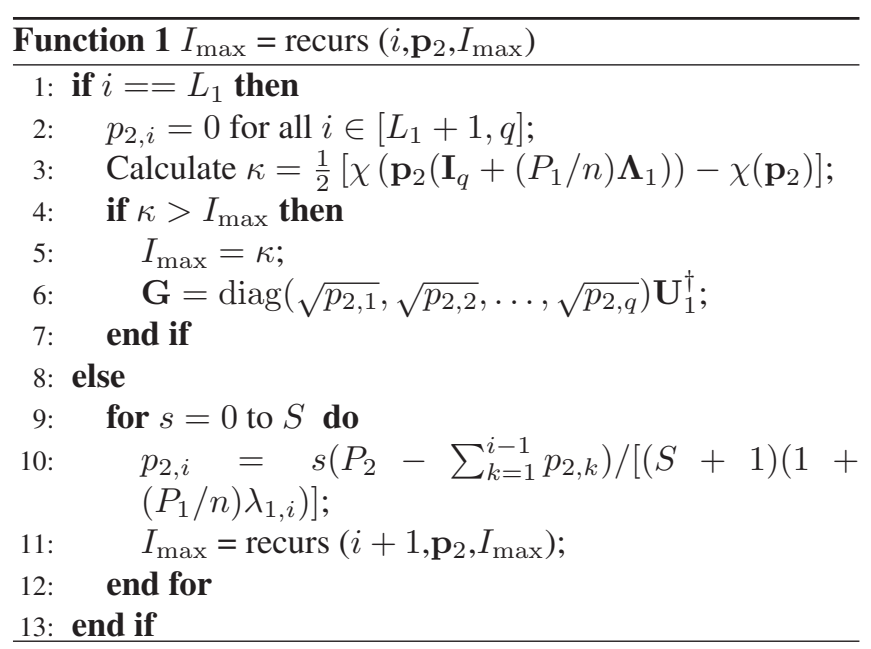

In the main algorithm, prior to use the function "recurs", $i$ and $I_{\max }$ must be set to one and zero, respectively. The complexity of such an algorithm increases with $q$ and the number of sample $S$, which is used to adjust the accuracy of the algorithm. Hence, this algorithm can be difficult to implement for very large $q$ and $S$ values, however it can be used as an upper benchmark (UB) for AF mutual information performance. Consequently, we develop a lower-complexity power allocation method, which is easy to implement, in the next section.

\subsection{Equal eigen mode power allocation method}

In our EEM power allocation method, the $\mathbf{G}$ matrix is designed to maximize $I\left(\mathbf{y}_{2} ; \mathbf{x}\right)$ by assuming that all eigen mode of $\mathbf{H}_{2}$ are equivalent. This method is a cunningly modified version of the methods propose in $[7,8]$ for the case where both S-R and R-D CSI, i.e., full CSI (FCSI), is known at the $\mathrm{R}$ node.

In the case of FCSI at the R node, $\mathbf{H}_{1}$ and $\mathbf{H}_{2}$ are known, and $\mathbf{H}_{2}$ can be decomposed via SVD as $\mathbf{H}_{2}=\mathbf{U}_{2} \widehat{\Lambda}_{2}^{\frac{1}{2}} \mathbf{V}_{2}^{\dagger}$, where $\mathbf{U}_{2} \in \mathbb{C}^{r \times r}$ and $\mathbf{V}_{2} \in \mathbb{C}^{q \times q}$ are unitary matrices, $\widehat{\boldsymbol{\Lambda}}_{2} \in \mathbb{C}^{r \times q}$ is a rectangular diagonal matrices, and $\boldsymbol{\Lambda}_{2}=$ $\widehat{\boldsymbol{\Lambda}}_{2}^{\frac{1}{2}} \widehat{\boldsymbol{\Lambda}}_{2}^{\frac{1}{2} \dagger}$ is a $r \times r$ diagonal matrix with diagonal elements $\lambda_{2, i} \in \mathbb{C}$, which are sorted in descending order as in [7]. Notice that $\lambda_{2, i} \neq 0$ for $i \in\left[1, L_{2}\right]$ and that $\lambda_{2, i}=0$ for $i \in\left[L_{2}+1, r\right]$, with $L_{2}=\min \{r, q\}$. Furthermore, $\mathbf{G}=$ $\mathbf{V}_{2} \widetilde{\mathbf{G}} \mathbf{U}_{1}^{\dagger}$, and consequently, we can re-express (3) after some simplifications as

$$
I\left(\mathbf{y}_{2} ; \mathbf{x}\right)=\frac{1}{2} \sum_{i=1}^{L} \log _{2}\left(\frac{p_{2, i} \lambda_{2, i}\left(1+\left(P_{1} / n\right) \lambda_{1, i}\right)}{1+p_{2, i} \lambda_{2, i}}\right),
$$

where $L=\min \left\{L_{1}, L_{2}\right\}$. In order to obtain the $p_{2, i}$ values that maximize (10), we must then solve the following concave optimization problem

$$
\begin{aligned}
& \max _{\mathbf{p}_{2}} \frac{1}{2} \sum_{i=1}^{L} \log _{2}\left(\frac{p_{2, i} \lambda_{2, i}\left(1+\left(P_{1} / n\right) \lambda_{1, i}\right)}{1+p_{2, i} \lambda_{2, i}}\right) \\
& \text { s.t. } p_{2, i} \geq 0 ; P_{R}: \sum_{i=1}^{L} p_{2, i}\left(1+\left(P_{1} / n\right) \lambda_{1, i}\right) \leq P_{2},
\end{aligned}
$$

The optimum solution is obtained by using Lagrange method, [11], as follows

$p_{2, i}=\left[\frac{-\left(2+p_{1, i} \lambda_{1, i}\right)+\sqrt{4 \mu p_{1, i} \lambda_{1, i} \lambda_{2, i}+\left(p_{1, i} \lambda_{1, i}\right)^{2}}}{2 \lambda_{2, i}\left(1+p_{1, i} \lambda_{1, i}\right)}\right]_{+}$,

where $[x]_{+}=\max \{0, x\}, p_{1, i}=\left(P_{1} / n\right), i \in[1, L]$, and $\mu$ is the Lagrange multiplier which moves the constraints in (12) into the maximization cost function $P_{R}$ of the optimization problem. The starting point for $\mu$ is

$$
\mu_{\min }=\max _{i \in[1, L]}\left\{\frac{1+\left(P_{1} / n\right) \lambda_{1, i}}{\left(P_{1} / n\right) \lambda_{1, i} \lambda_{2, i}}\right\}
$$

and $\mu$ can be updated by using the Newton-Raphson method [12] until $\mu^{\star}$ is obtained. Let the function

$$
f(\mu)=\sum_{i=1}^{L} p_{2, i}(\mu)\left(1+\left(P_{1} / n\right) \lambda_{1, i}\right)-P_{2},
$$

where $p_{2, i}(\mu)$ is given in (12), then $\mu^{\star}$ is chosen such that it fulfills the following inequality $f\left(\mu^{\star}\right)<\epsilon$, with $\epsilon \ll 1$.

Concerning the case where only S-R link CSI is available at the R node, $I\left(\mathbf{y}_{2} ; \mathbf{x}\right)$ can be expressed as in (6) with $\mathbf{G}=$ $\widetilde{\mathbf{G}} \mathbf{U}_{1}^{\dagger}$ instead of $\mathbf{G}=\mathbf{V}_{2} \widetilde{\mathbf{G}} \mathbf{U}_{1}^{\dagger}$ in the FCSI case. Since $\mathbf{R}$ does not have any information about $\lambda_{2, i}$ as well as $\mathbf{V}_{2}$, in our approach, we assume that $\mathbf{V}_{2}=\mathbf{I}_{q}$ and that all the eigen modes of $\mathbf{H}_{2}$ are equal to one, i.e., $\lambda_{2, i}=1$ for all $i \in\left[1, L_{2}\right]$. As a result, the optimization problem in (11) is converted to 


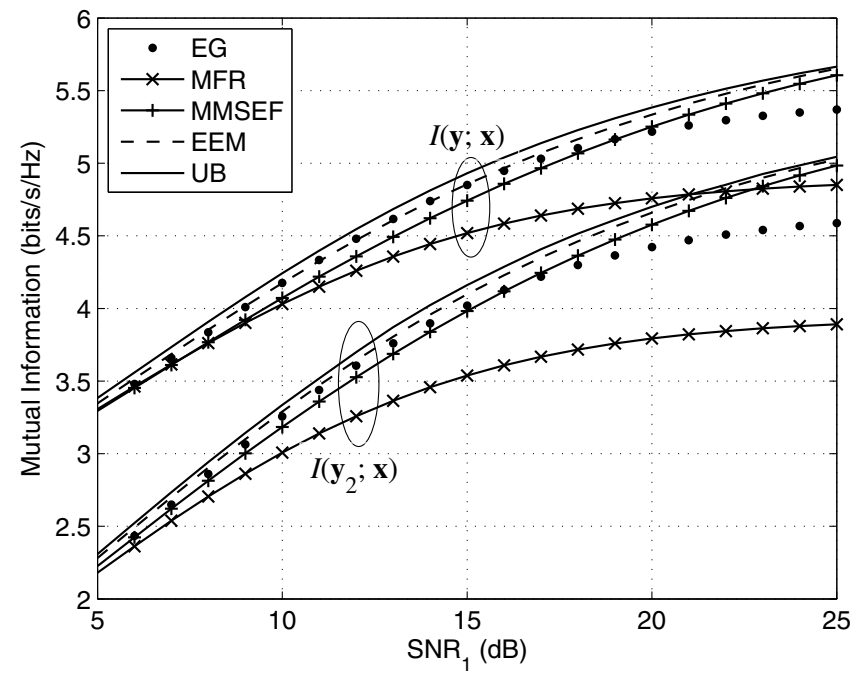

Fig. 2. Non-cooperative, $I\left(\mathbf{y}_{2} ; \mathbf{x}\right)$, and Cooperative, $I(\mathbf{y} ; \mathbf{x})$, Mutual information performance of various power allocation methods for $n=q=r=4, \mathrm{SNR}_{0}=0 \mathrm{~dB}$ and $\mathrm{SNR}_{2}=10 \mathrm{~dB}$.

$$
\begin{aligned}
& \frac{1}{2} \max _{\mathbf{p}_{2}} \sum_{i=1}^{L} \log _{2}\left(\frac{p_{2, i}\left(1+\left(P_{1} / n\right) \lambda_{1, i}\right)}{1+p_{2, i}}\right) \\
& \text { s.t. } p_{2, i} \geq 0 ; P_{R}: \sum_{i=1}^{L} p_{2, i}\left(1+\left(P_{1} / n\right) \lambda_{1, i}\right) \leq P_{2},
\end{aligned}
$$

and the optimum solution to this problem is given as in (12) but where $\lambda_{2, i}=1$. Our novel power allocation method can be summarized as follows

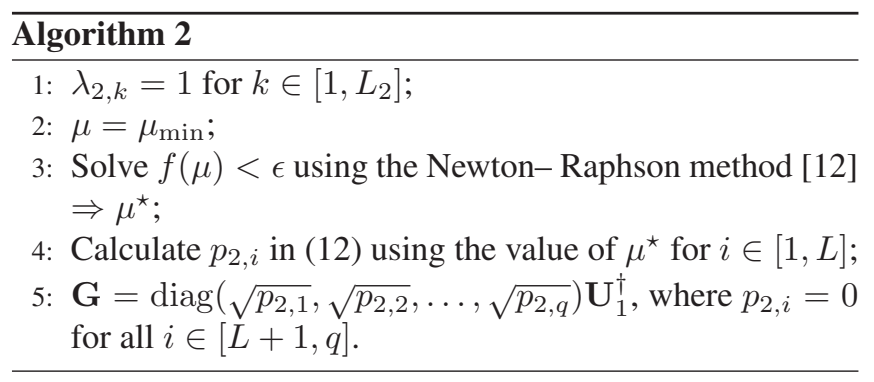

\section{NUMERICAL RESULTS}

The two novel power allocation methods introduced in Section 3, i.e., the analytical method in Section 3.2 and the EEM method in Section 3.3, are compared against the EG, MFR and MMSEF methods, in terms of mutual information and BER performance for various number of transmit/receive antennas as well as different signal-to-noise ratio (SNR) settings.

In our simulations, we denote $\mathrm{SNR}_{0}$ as the $\mathrm{SNR}$ of the S-D link, $\mathrm{SNR}_{1}$ as the SNR of the S-R link and $\mathrm{SNR}_{2}$ as the SNR of the R-D link. Moreover, we have set $S=10$ for the analytical UB method and we have used the Matlab function

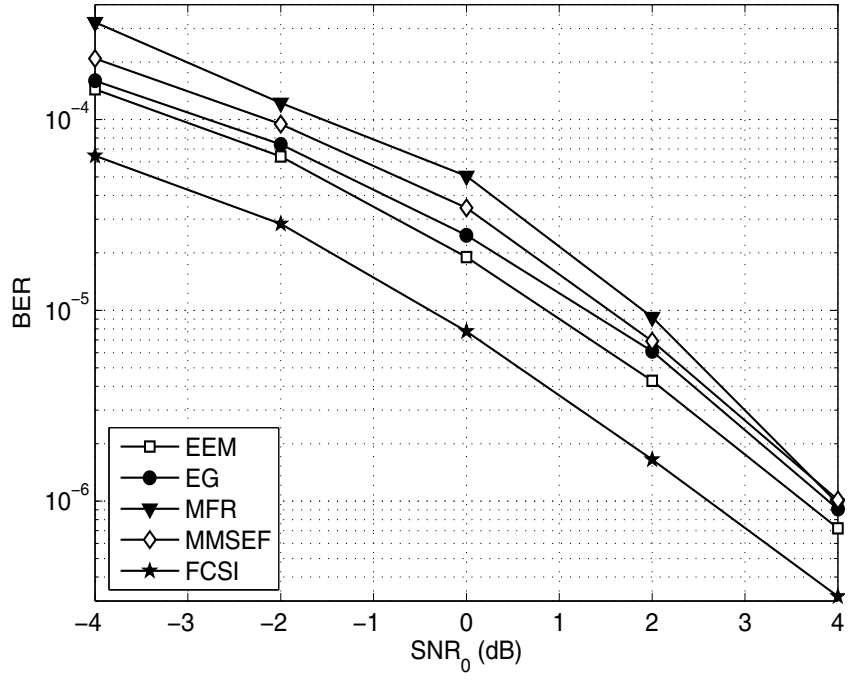

Fig. 3. Cooperative BER performance of various power allocation methods for $n=q=r=2, \mathrm{SNR}_{1}=10 \mathrm{~dB}$ and $\mathrm{SNR}_{2}=15 \mathrm{~dB}$

"solve" in order to obtain the root $d_{0}$ of $P(d)$ in (8). For the EEM method, we have set $\epsilon<1.10^{-5}$.

In the BER performance evaluation, at the source, we rely on a bit interleaved coded modulation (BICM) model where the information bit sequences are encoded with an optimum half-rate systematic recursive convolutional code with generator polynomial $(13,15)$ in octal representation. Then, the interleaved coded bits are QPSK modulated and transmitted directly over transmit antennas to the other nodes. At the destination an optimum maximum a posteriori (MAP) detector is used to extract transmitted information from the $\mathrm{S}$ node. A single-tap independent and identically distributed (i.i.d) Rayleigh fading channel is assumed between the various links, S-D, S-R, and R-D.

In Fig. 2, we compare the cooperative and non-cooperative mutual information performance of the various power allocation methods described in Section 3, for $n=q=r=4$, $\mathrm{SNR}_{0}=0 \mathrm{~dB}$ and $\mathrm{SNR}_{2}=10 \mathrm{~dB}$. First, the results confirm that our UB method outperforms all the other methods and hence provides an UB for AF mutual information performance. Moreover, our novel EEM method performs as good as EG for low and medium values of $\mathrm{SNR}_{1}$ and yet far better than EG for high $\mathrm{SNR}_{1}$ values. Our EEM method also outperforms MMSEF and MFR for any $\mathrm{SNR}_{1}$ values.

In Figs. 3 and 4, we present the cooperative BER performance of the various power allocation methods described in Section 3 as well as the FCSI method [7,8], for $n=q=$ $r=2$ and $n=q=r=4$, respectively, and considering $\mathrm{SNR}_{1}=10 \mathrm{~dB}$ and $\mathrm{SNR}_{2}=15 \mathrm{~dB}$. The graphs indicate that the EEM method outperforms all the other traditional techniques including EG, MFR and MMSEF, when only receive CSI is available at the relay. The MFR method exhibits 


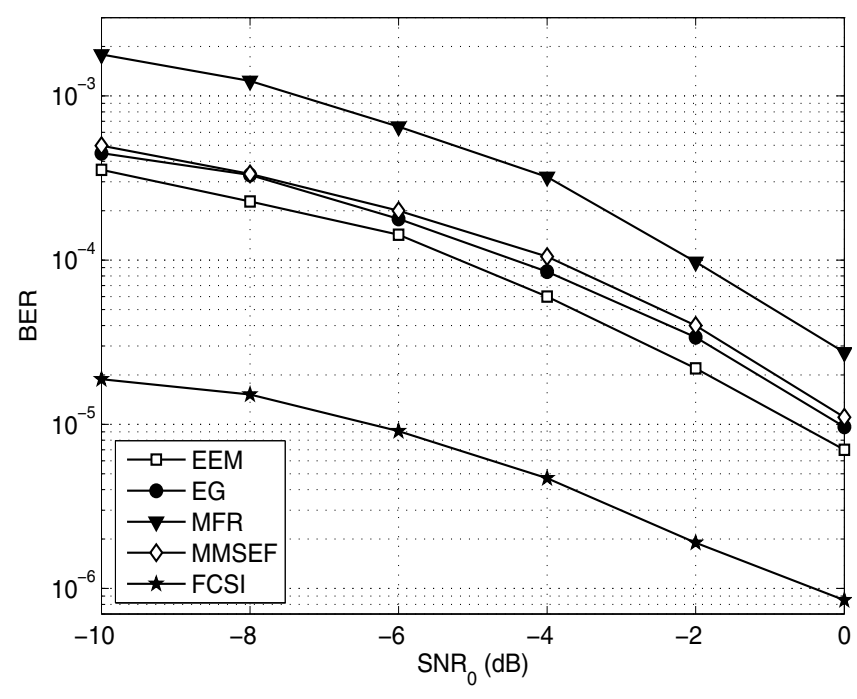

Fig. 4. Cooperative BER performance of various power allocation methods for $n=q=r=4, \mathrm{SNR}_{1}=10 \mathrm{~dB}$ and $\mathrm{SNR}_{2}=15 \mathrm{~dB}$

the worst performance compared to the others. As a benchmark, the BER performance of the FCSI method is also displayed. This result shows the performance improvement that the knowledge of the transmit CSI at the relay can bring to the system, but at the cost of a higher complexity compared to the EEM method. Similar behaviour can be observed for $n=q=r=4$, but with larger performance difference between the various power allocation techniques, especially, between the FCSI method and the receive CSI only techniques. In this $\mathrm{SNR}$ setting, given the fact that $\mathrm{SNR}_{1}$ and $\mathrm{SNR}_{2}$ are fairly high in comparison with $\mathrm{SNR}_{0}$, the performance of the direct communication is worse than the one of cooperative communication, and consequently, the direct communication performance has not been plotted here.

\section{CONCLUSION}

In this paper, two novel power allocation methods for nonregenerative cooperative MIMO communication have been proposed. Both these methods are based on the maximization of the mutual information of the relay link when only receive CSI is available at the relay. The first method is an analytical solution which maximizes the expectation of the mutual information, and it has been implemented via a highcomplexity recursive algorithm. The second method is solving a concave optimization problem with a low-complexity of implementation. Numerical results presented in this paper have shown that both methods outperform other common relay receive CSI only power allocation techniques in terms of mutual information and BER. In the future, novel power allocation techniques for the case where the transmit CSI is known at the $\mathrm{S}$ node will be investigated.

\section{REFERENCES}

[1] J. N. Laneman and G. W. Wornell, "Distributed SpaceTime-Coded Protocols for Exploiting Cooperative Diversity in Wireless Networks," IEEE Trans. Inform. Theory, vol. 49, no. 10, pp. 2415-2425, Oct. 2003.

[2] A. Sendonaris, E. Erkip, and B. Aazhang, "User Cooperation Diversity Part I - System Description," IEEE Trans. Commun., vol. 51, no. 11, pp. 1927-1938, Nov. 2003.

[3] A. Sendonaris, E. Erkip, and B. Aazhang, "User Cooperation Diversity Part II - Implementation Aspects and Performance Analysis," IEEE Trans. Commun., vol. 51, no. 11, pp. 1939-1948, Nov. 2003.

[4] M. Janani, A. Heday, T. Hunter, and A. Nosratinia, "Coded Cooperation in Wireless Communications: Space-Time Transmission and Iterative Decoding," IEEE Trans. Signal Processing, vol. 52, no. 2, pp. 362-371, Feb. 2004.

[5] A. Nosratinia, T. E. Hunter, and A. Hedayat, "Cooperative Communicationin Wireless Networks," IEEE Commun. Mag., vol. 42, no. 10, pp. 74-80, Oct. 2004.

[6] J. N. Laneman, D. N. C. Tse, and G. W. Wornell, "Cooperative Diversity in Wireless Networks: Efficient Protocols and Outage Behavior," IEEE Trans. Inform. Theory, vol. 50, no. 12, pp. 3062-3080, Dec. 2004.

[7] O. Muñoz-Medina, J. Vidal, and A. Agustn, "Linear Transceiver Design in Nonregenerative Relays with Channel State Information," IEEE Trans. Signal Processing, vol. 55, no. 6, pp. 2593-2604, June 2007.

[8] Y. Fan and J. Thompson, "MIMO Configurations for Relay Channels: Theory and Practice," IEEE Trans. Wireless Commun., vol. 6, no. 5, pp. 1774-1786, May 2007.

[9] T. M. Cover and J. A. Thomas, Elements of Information Theory, ed. Wiley, New-York, USA, 1991.

[10] F. Héliot, R. Hoshyar, and R. Tafazolli, "An Asymptotical Approximation of Outage Probability for Distributed MIMO Systems," in ICT-mobile summit, Santander, Spain, June 2009.

[11] S. Boyd and L. Vandenberghe, Convex Optimization, Cambridge Univ. Press, Cambridge, UK, 2004.

[12] "Wolfram Mathworld Website," [Online]. Available: http://mathworld.wolfram.com/NewtonsMethod.html. 